\title{
The vacuum bell for treatment of pectus excavatum: an alternative to surgical correction?
}

\author{
Frank-Martin Haecker*, Johannes Mayr \\ Department of Pediatric Surgery, University Children's Hospital, P.O. Box, CH 4005 Basel, Switzerland
}

Received 30 August 2005; received in revised form 3 January 2006; accepted 10 January 2006; Available online 13 February 2006

\begin{abstract}
Objective: Pectus excavatum (PE) is the most common chest wall malformation and one of the most frequent major congenital anomalies. The surgical repair of PE in childhood is a well-established procedure. Previously used operative techniques to correct PE were largely based on the Ravitch technique. Today, the minimally invasive repair (MIRPE) by Nuss is well established. Conservative treatment with the vacuum bell to elevate the funnel in patients with PE represents a potential alternative. Methods: A suction cup is used to create a vacuum at the anterior chest wall. A patient-activated hand pump is used to reduce the pressure up to $15 \%$ below atmospheric pressure. Three different sizes of vacuum bell exist which are selected according to the individual patients age. When creating the vacuum, the lift of the sternum is obvious and remains for a different time period. The device should be used for a minimum of $30 \mathrm{~min}$ ( 2 per day), and may be used up to a maximum of several hours daily. Presently, a 12-15-month course of treatment is recommended. In addition, the device was used intraoperatively during the MIRPE procedure to enlarge the retrosternal space to ensure safer passage of the introducer in a few patients. Results: Thirty-four patients ( 31 males, 3 females), aged 6-52 years (median 17.8 years) used the vacuum bell for 1 to maximum 18 months (median 10.4 months). Follow-up included photography and clinical examination every 3 months. Computed tomographic scans showed that the device lifted the sternum and ribs immediately. In addition, this was confirmed thoracoscopically during the MIRPE procedure. After 3 months, an elevation of more than $1.5 \mathrm{~cm}$ was documented in 27 patients (79\%). After 12 months, the sternum was lifted to a normal level in five patients (14.7\%). Relevant side effects were not noted. Conclusions: The vacuum bell has proved to be an alternative therapeutic option in selected patients with PE. The initial results proved to be dramatic, but long-term results are so far lacking, and further evaluation and follow-up studies are necessary. In addition, the method may assist the surgeon during the MIRPE procedure.
\end{abstract}

(C) 2006 Elsevier B.V. All rights reserved.

Keywords: Pectus excavatum; Conservative treatment; Vacuum bell

\section{Introduction}

Pectus excavatum (PE) is the most common chest wall malformation and one of the most frequent major congenital anomalies, occurring in approximately 1 in every 300 births [1]. The defect is noticeable at birth in more than $85 \%$ of infants, whereas a later onset is observed in patients with Marfan syndrome. Until 10 years ago, operations to correct PE deformities were largely based on the technique described by Ravitch [2]. In 1998, the technique of minimally invasive repair of PE (MIRPE) was first described by Nuss et al. [3] to avoid several operative features of the modified Ravitch repair procedure. By inserting a convex steel bar under the sternum through a small lateral thoracic incision without rib incision or resection, it was possible to correct even a severe degree of PE in prepubertal patients. This procedure avoids features of the Ravitch technique such as anterior chest

\footnotetext{
* Corresponding author. Tel.: +4161 6855239; fax: +41616855011.

E-mail address: frankmartin.haecker@ukbb.ch (F.-M. Haecker).
}

incision, resection of costal cartilages and sternal osteotomy. The shorter operating time, smaller incisions and considerably less dissection has made the MIRPE procedure very appealing both to surgeons and patients, thereby resulting in a large increase of the number of patients requesting operative treatment, and consequently in an increase of the number of PE repairs. However, with the widespread use of the MIRPE procedure, the character and number of complications has increased [4-7]. Additionally, in many cases of PE, the degree of pectus deformity does not immediately warrant surgery, yet patients may benefit from some type of nonsurgical treatment. Other patients are reluctant to undergo surgery because of the pain associated with postoperative recovery and the risk of imperfect results. Due to these facts, the introduction of the vacuum bell for conservative treatment of $\mathrm{PE}$ has made this alternative therapy a focus of interest of patients.

The procedure of applying a vacuum to elevate the sternum was first used more than 100 years ago [8]. Despite the risks and unsatisfactory results after operative therapy for some patients, there has been little progress in the 
therapeutic use of the vacuum therapy during the last few decades. In the meantime, materials have improved and the vacuum devices can now exert strong forces. We report our preliminary experience using such a vacuum bell for conservative treatment of PE.

\section{The vacuum bell}

A suction cup is used to create a vacuum at the chest wall. A vacuum up to $15 \%$ below atmospheric pressure is created by the patient using a hand pump (Fig. 1). Three different sizes $(16 \mathrm{~cm}, 19 \mathrm{~cm}$ and $26 \mathrm{~cm}$ in diameter) exist allowing selection according to the individual patients age. The development of a specific fitted model suitable for women is in progress. Pilot studies performed by Bahr and Schier in Jena, Germany (http://www.trichterbrust.de) showed that the device lifted the sternum and ribs immediately (Fig. 2). In addition, this was confirmed thoracoscopically during the MIRPE procedure. According to the user instructions and our experience, the vacuum bell should be used for a minimum of 30 min, twice per day, and may be used up to a maximum of several hours daily.

Complications and relevant side effects include subcutaneous haematoma, petechial bleeding, dorsalgia and transient paresthesia of the upper extremities during the application as well as rib fractures in rare cases. Contraindications of the method comprise skeletal disorders such as osteogenesis imperfecta and Glisson's disease, vasculopathies (e.g. Marfan's syndrome, abdominal aneurysm), coagulopathies and cardiac disorders. To exclude these disorders, a standardised evaluation protocol was routinely performed before beginning the therapy.

\section{Patients and methods}

Thirty-four patients ( 31 males, 3 females), aged from 6 to 52 years (median 17.8 years, Fig. 3) were treated with the

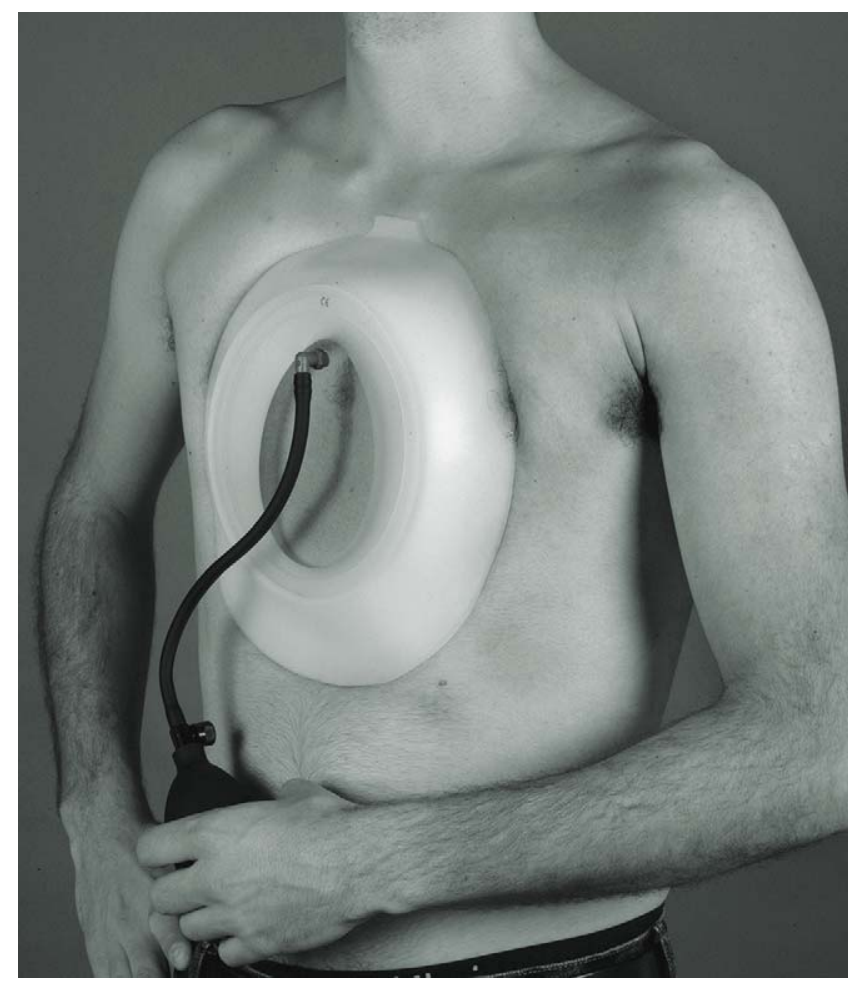

Fig. 1. Application of the vacuum bell (23-year-old patient).

vacuum bell for 1 to a maximum of 18 months (median 10.4 months). For further evaluation, patients were divided into two groups. Group 1 included 23 paediatric patients aged $\leq 18$ years. Group 2 comprised 11 adult patients aged from 19 to 52 years (Fig. 3). Standardised evaluation before starting the procedure included 3D computerised tomography (CT) scan, pulmonary function tests, cardiac evaluation with electrocardiogram and echocardiography and photo documentation. In addition, the depth of PE was measured. Patients underwent follow-up at 3-6 monthly intervals including photography and clinical examination.

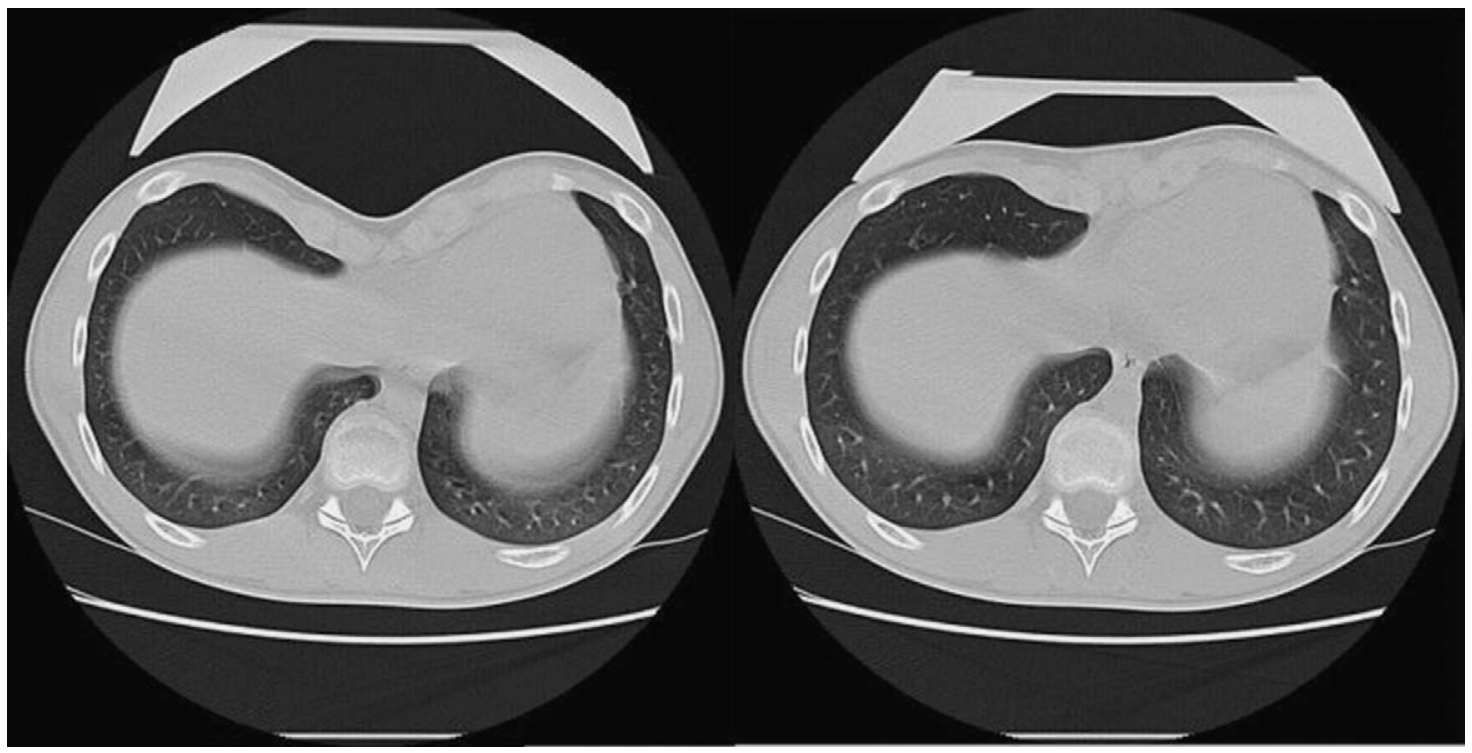

Fig. 2. CT-scan before and after 2 min of vacuum (Bahr, http://www.trichterbrust.de). 


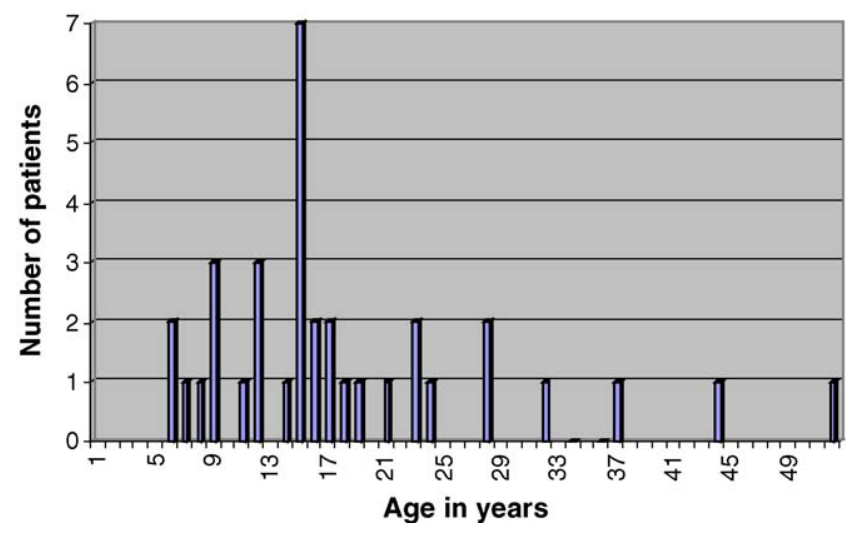

Fig. 3. Age distribution.

The first application of the vacuum bell occurred under supervision of the attending doctor. The length of time of daily application of the vacuum bell varied widely between patients. Patients were told to follow the user instructions applying the device twice daily for 30 min each. In fact, the duration and frequency of daily application depends on the patients individual decision and motivation. One patient, a 52-year-old businessman, used the vacuum bell 4-6 h daily during office hours. Two adolescent boys applied the device every night for 7-8 h. A 21-year-old male patient, who underwent PE repair by the Ravitch procedure 6 years ago, was dissatisfied with the postoperative result and recently started treatment with the vacuum bell. In addition to the daily application of the device, all patients were recommended to carry on undertaking physiotherapy as well as sports, e.g. swimming. Physical fitness, especially chest fitness with well developed pectoralis major muscle did not hinder the correction in anyone of our patients.

\section{Results}

During the first $1-5$ applications, all patients experienced moderate pain in the sternum and reported a feeling of uncomfortable pressure within the chest. Adolescent and older patients developed moderate subcutaneous haematoma, which disappeared within a few hours. Two patients reported recurrent transient paresthesia of the upper extremities during the application. But this phenomena disappeared when lower atmospheric pressure was used during application. One 45-year-old patient suffering from recurrent dorsalgia, reducing the application time, prevented the occurrence of discomfort. Analgesic medication was not necessary in any patient. In patients younger than 10 years of age, the application was supervised by the parents. In these patients, there were no relevant side effects reported.

Before starting the treatment, the depth of $\mathrm{PE}$ ranged from $2.5 \mathrm{~cm}$ to $5 \mathrm{~cm}$. In all patients, the sternum and the ribs were lifted immediately after application of the device. The elevation of the sternum was more obvious in the paediatric group. However, after removal of the vacuum bell the sternum subsided faster in paediatric patients than in adults, where the elevation lasted for 30-60 min. In accordance with this observation, the elevation of the sternum was more successful within the first 6-9 months of application in group 1. In contrary, adult patients demonstrated a slower but continuous decrease of PE. In 27 patients (79\%), after 3 months of treatment, a permanent elevation of more than $1.5 \mathrm{~cm}$ was documented. Figs. 4 and 5 demonstrate the result after 6 months and after 10 months treatment, respectively. In five patients $(14.7 \%)$, the sternum was permanently lifted to a normal level after 12 months. In three patients with asymmetric PE, the depth of PE has decreased after 9 months, but the asymmetry is still visible. A 19-year-old patient with an isolated, asymmetric deformity of the upper chest wall stopped the application after 6 months due to an unsatisfactory result. At follow-up, all paediatric and adult patients except the last one were satisfied and expressed their motivation to continue the application.

\section{Discussion}

Nowadays information on new therapeutic modalities circulates not only among surgeons and paediatricians but also rapidly among patients. In particular, patients who refused operative treatment by previously available procedures, now appear at the outpatient clinic and request to be considered for the new method. The vacuum method was used as early as 1910 by Lange [8] for elevating the sternum, but has not been previously applicated in a large number of patients, and long-term results are not reported. The vacuum bell used in our patients group was developed by an engineer, who himself suffered from PE (Klobe, http: / / www.trichterbrust.de). Long-term evidence of persistent effects of the

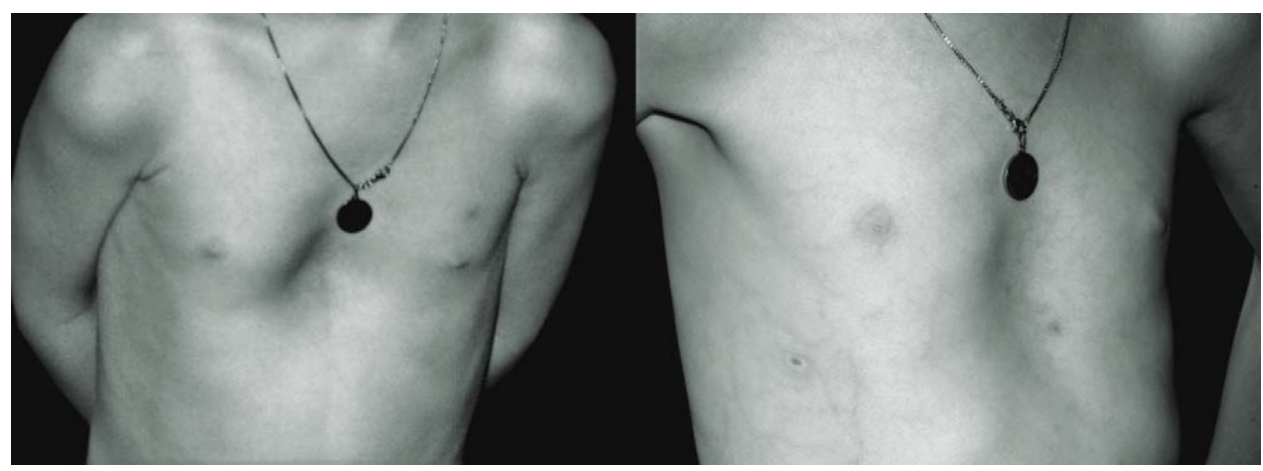

Fig. 4. Six-year-old boy, before (left: depth of $P E=3.3 \mathrm{~cm}$ ) vacuum bell therapy and after 6 months (right: depth of $P E=1.1 \mathrm{~cm})$, 3 days after the last application. 


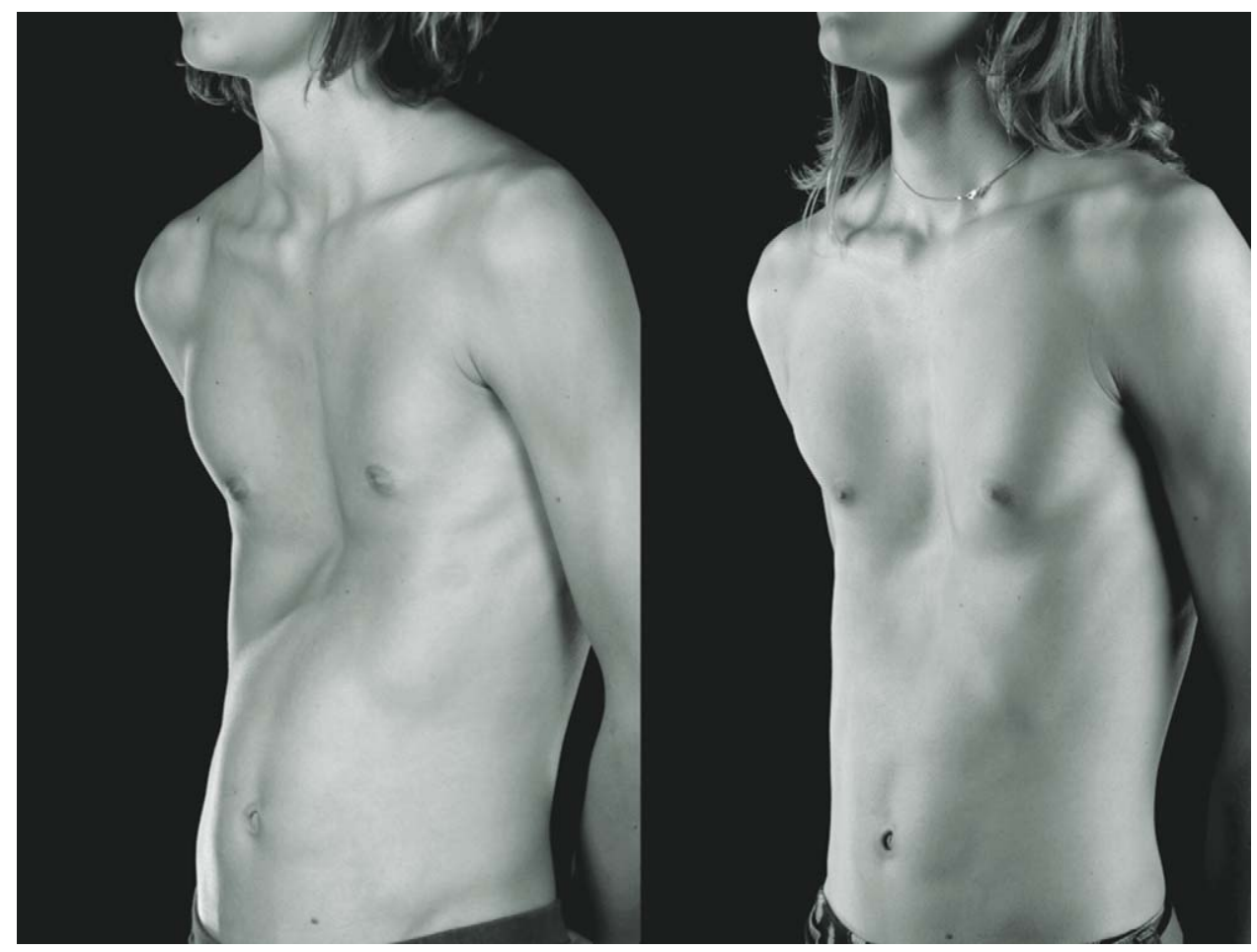

Fig. 5. Sixteen-year-old boy, before (left: depth of PE $=4.5 \mathrm{~cm}$ ) vacuum bell therapy and after 10 months (right: depth of PE $=2.0 \mathrm{~cm}$ ), $1 \mathrm{week}$ after the last application.

treatment modality are not yet available, since the method has only been used for a maximum of 2 years. However, initial results proved dramatic, and the acceptance and compliance of patients seem to be good. In many cases of PE, the degree of pectus deformity does not immediately warrant surgery, yet patients may benefit from some type of nonsurgical treatment. Other patients are disinclined to undergo surgery because of possible complications after surgery, because of the pain associated with postoperative recovery and the risk of imperfect results. Thus, the introduction of the vacuum bell for conservative treatment of PE has generated much interest among patients with PE, despite of the lack so far of long-term results of the method.

The success of a therapeutic procedure not only requires a good technique, but also depends on a appropriate indication. In our study, patients, who presented with symmetric and mild PE, seemed to show a more successful outcome than those with asymmetric and deep PE. The application of the vacuum bell was well tolerated by both paediatric and adult patients. All patients except one were satisfied with the use of the vacuum bell, although objectively assessed improvement of PE varied between the individuals. All our patients were recommended to carry on undertaking sports and physiotherapy, so that the accompanying improvement of body control was an important factor in outcome. The participation of patients themselves in the 'active' treatment of PE clearly increases motivation to maintain therapy. The manufacturers instructions and our treatment protocol recommended application of the device twice daily for $30 \mathrm{~min}$ each. However, the definitive duration and length of use was determined by the individual patient and the parents, respectively. As demonstrated in the CT-scan (Bahr, http://www.trichterbrust.de), the force of the vacuum bell is strong enough to deform the chest within minutes.
Therefore, especially in children younger than 10 years of age the application of the vacuum bell has to be performed carefully and should be supervised by an adult.

When creating the vacuum, the elevation of the sternum is obvious and persists for a distinct period of time. Therefore, the vacuum cup may also be useful in reducing the risk of injury to the heart during the MIRPE procedure, where the riskiest step of the procedure is the advancement of the introducer between the heart and sternum. Since the manufacturer of the device has not yet a license to sterilise the vacuum bell, this additional use has to be considered as a clinical trial. In accordance with our hospital hygienist, we applied the vacuum bell during the MIRPE procedure in a few patients with good experience. In addition, the vacuum bell may be useful in a way of 'pretreatment' to surgery. Since none of the 34 patients asked for the MIRPE procedure, we could not confirm this hypothesis at the moment.

In conclusion, the vacuum bell may allow some patients with PE to avoid surgery. Especially patients with symmetric and mild PE may benefit from this procedure. However, the time of follow-up in our series is too short to confirm this with any certainty. Additionally, the intraoperative use of the vacuum bell during the MIRPE may facilitate the introduction of the pectus bar. This must be evaluated by further studies. In any case, the method seems to be a valuable adjunct therapy in the treatment of PE.

\section{References}

[1] Molik KA, Engum SA, Rescorla FJ, West KW, Scherer LR, Grosfeld JL. Pectus excavatum repair: experience with standard and minimal invasive techniques. J Pediatr Surg 2001;36:324-8.

[2] Ravitch MM. The operative treatment of pectus excavatum. Ann Surg 1949;129:429-44. 
[3] Nuss D, Kelly RE, Croitoru DP, Katz ME. A 10-year review of a minimally invasive technique for the correction of pectus excavatum. J Pediatr Surg 1998;33:545-52.

[4] Croitoru DP, Kelly RE, Goretsky MJ, Lawson ML, Swoveland B, Nuss D. Experience and modification update for the minimally invasive Nuss technique for pectus excavatum repair in 303 patients. J Pediatr Surg 2002;37:437-45.

[5] Haecker F-M, Bielek J, von Schweinitz D. Minimally invasive repair of pectus excavatum (MIRPE): the basel experience. Swiss Surg 2003;9:289-95.
[6] Berberich T, Haecker F-M, Kehrer B, Erb T, Günthard J, Hammer J, Jenny P. Postcardiotomy syndrome after minimally invasive repair of pectus excavatum. J Pediatr Surg 2004;39:e1-0.

[7] Van Renterghem KM, von Bismarck S, Bax NMA, Fleer A, Hoellwarth M. Should an infected Nuss bar be removed? J Pediatr Surg 2005;40: 670-3.

[8] Lange F. Thoraxdeformitäten. In: Pfaundler M, Schlossmann A, editors. Handbuch der Kinderheilkunde, vol. V. Chirurgie und Orthopädie im Kindesalter. Leipzig: FCW Vogel; 1910. p. 157. 